\title{
Globe
}

Revue internationale d'études québécoises

\section{Redessiner la cartographie des écritures migrantes}

\section{Gilles Dupuis}

Volume 10, numéro 1, 2007

URI : https://id.erudit.org/iderudit/1000083ar

DOI : https://doi.org/10.7202/1000083ar

Aller au sommaire du numéro

Éditeur(s)

Globe, Revue internationale d'études québécoises

ISSN

1481-5869 (imprimé)

1923-8231 (numérique)

Découvrir la revue

Citer cette note

Dupuis, G. (2007). Redessiner la cartographie des écritures migrantes. Globe, 10(1), 137-146. https://doi.org/10.7202/1000083ar d'utilisation que vous pouvez consulter en ligne.

https://apropos.erudit.org/fr/usagers/politique-dutilisation/ 


\title{
NOTE CRITIQUE
}

REDESSINER

\section{LA CARTOGRAPHIE DES ÉCRITURES MIGRANTES}

\author{
GILLES DUPUIS \\ Université de Montréal
}

Depuis la parution de la première histoire de la littérature migrante au Québec ${ }^{1}$, d'un dictionnaire des écrivains émigrés ${ }^{2}$, et de deux essais portant sur des problématiques connexes - la littérature d'exil et la

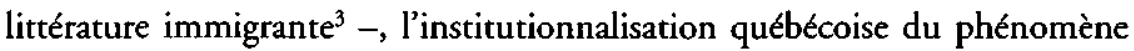
des "écritures migrantes", amorcée dès la fin des années 1980 avec les travaux pionniers de Pierre Nepveu ${ }^{4}$ et de Simon Harel's, s'est pour ainsi dire parachevée. On ne compte plus les publications savantes, sous forme de monographies, d'ouvrages collectifs, de dossiers de revues et d'articles isolés, qui se penchent sur le corpus migrant d'un point de vue historique, littéraire, sociologique, pédagogique, etc. Si certains travaux sont animés par des motifs

$$
4+4
$$

1. Clément MOISAN et Renate HILDEBRAND, Ces étrangers du dedans. Une histoine de l'écrirure mıgrante au Québec (1937. 1997), Québec, Nota bene, 2001.

2. Daniel CHARTER, Dictronnaire des tecrivains tmigrés au Québec 1800-1999, Québec, Nota bene, 2003.

3. Silvie BERNIER, Les hértsers d'Ulysse, Outremont, Lanctôt, 2002; Nathalie PRUD'HOMME, La problematique identité collective et les litteratures (tm) migrantes au Quebec. Mona Latif Ghattas, Antonio D'Alfonso et Marco Micone, Quebec, Nota bene, 2002. Voir aussi mon compre-rendu de ces deux ouvrages dans Spirale, $n^{\circ} 193$, novembre-décembre 2003, p. 22 23.

4. Pierre NEPVEU, L'écologie du réel. Mort et nassance de La litterature québécoise contemporaine, Montréal, Boréal, 1988. 5. Simon HAREL, Le voleur de parcours, Longueuil, Le Préambule, 1989. 
en partie personnels (pensons aux contributions originales de Régine Robin et de Sherry Simon), la plupart sont guidés par un souci d'objectivité que commande le milieu universitaire auquel ils se destinent. De ce nombre, un important contingent provient de l'extérieur du Québec, notamment de l'Europe non francophone (Allemagne, Autriche, Italie, Espagne), comme en témoigne le dossier de ce numéro dirigé par une spécialiste de la littérature migrante, Carmen Mata Barreiro de l'Université Autonome de Madrid. Les études qui s'intéressent à la question de l'autre et de l'étranger, de l'identité et de l'identitaire, en relation avec le territoire réel ou imaginaire, sont aussi légion. Le groupe de recherche "Le soi et l'autre" de l'UQAM, qui a donné lieu à une collection chez VLB dirigée par Pierre Ouellet, accueille chaque année de nouveaux titres tout aussi pertinents pour ce numéro de la revue Globe.

En syntonie avec le thème de son dossier, "Étranger et territorialité", il m'est apparu judicieux dans cette note critique qui l'accompagne de me concentrer principalement sur deux livres parus récemment dans le sillon des études portant sur la littérature migrante, mais qui adoptent des points de vue opposés, voire contradictoires, reflétant bien la double approche "objective" et "subjective" du sujet: un ouvrage didactique de Monique Lebrun et Luc Collès (avec la participation de Marie-Cécile Robinet $)^{6}$ et un essai polémique de Simon Harel 7 . Le premier a le mérite de replacer la littérature migrante au Québec dans un contexte plus large, soit l'espace francophone qui lui reste souvent étranger malgré la proximité de la langue. Le second permet d'amorcer une critique de cette littérature et de sa reconnaissance institutionnelle à partir d'une redéfinition des notions de territoire et d'identité. Je situerai l'étude panoramique de Lebrun et Collès dans le prolongement de l'historique que Clément Moisan et Renate Hildebrand dressent de la littérature migrante au Québec; j'élargirai par la suite la critique que fait Harel de l'écriture migrante au concept de «braconnage" qu'il propose dans son essai ultérieur ${ }^{8}$.

\section{$+4$}

6. Monique LEBRUN et Luc COLLES, La littrature migrante dans l'espace francophone. Belgique - France - Qutbec - Susse, Cortil-Wodon, E.M.E. \& InterCommunications S.P.R.L., 2007.

7. Simon HAREL, Les passages obligés de l'écriture migrante, Montréal, XYZ, 2005.

8. Sumon HAREL, Braconnages identitaires. Un Quebec palempseste. Montréal, VLB, 2006. Voir mon compte-rendu de cet essai paru dans Spirale, $\mathrm{n}^{\circ} 211$, novembre-décembre 2006, p. 41-42. 


\section{UNE LITTERATURE MIGRANTE HORS QUEBEC}

Depuis l'introduction de l'expression "écritures migrantes» au Québec expression que l'on a souvent attribuée au poète d'origine haïtienne Robert Berrouët-Oriol', alors qu'elle se laisse au moins retracer à un autre écrivain de même origine, Émile Ollivier ${ }^{10}$ - la critique s'entendait pour voir dans ce qu'elle désignait un phénomène propre à la littérature québécoise. La publication simultanée des romans La Québécoite de Régine Robin et Le piano-trompette de Jean Basile en 1983, date charnière où commence à paraître à Montréal la revue transculturelle Vice versa qui va rapidement prendre la relève de la revue interculturelle Dérives, a contribué rétrospectivement à consacrer le phénomène "migrant" comme un courant littéraire spécifiquement québécois. S'il est vrai que c'est ici que le concept a connu sa plus grande fortune critique, au point où le Québec peut s'enorgueillir d'avoir pavé la voie à la reconnaissance institutionnelle de la littérature migrante, le premier mérite de l'ouvrage de Lebrun et Collès (et non le moindre) est de nous montrer que cette littérature se pratiquait simultanément dans d'autres aires de la francophonie. Des romans comme La Pérégrine ${ }^{11}$ de Mireille Kuttel ou Éloge du migrant ${ }^{12}$ d'Adrien Pasquali, deux auteurs suisses issus de l'immigration italienne (donc non helvètes à l'origine), ressemblent étrangement, au-delà du titre évocateur, à ce que des écrivains comme Régine Robin et Marco Micone, et plus tard Abla Farhoud, ont pu écrire au Québec autour du même sujet.

Que ce soit en Belgique, avec l'apport original des communautés italienne et maghrébine; en France, avec l'importante littérature "beure"; ou en Suisse, avec l'émergence d'une littérature migrante "marginale" par rapport aux quatre littératures "autochtones», le phénomène socioculturel de la migrance est désormais bien attesté dans l'espace francophone, du moins européen. Car contrairement à ce qui se passe dans le monde anglo-saxon, notamment en Afrique du Sud, les auteurs ne se questionnent pas sur l'existence possible d'une littérature migrante d'origine européenne (ou d'autre provenance) écrite en français dans les anciennes colonies belges et françaises, voire dans la francophonie au sens large du terme. Tout écrivain français ou belge ayant écrit en français dans une ex-colonie (l'écrivain piednoir, par exemple) sera automatiquement rattaché à son "pays d'origine"

$$
+\div
$$

9. Robert Berroutet-ORIOL, "Effet d'exil n, Vice versa, n 17, décembre 1986-janvier 1987, p. 20-21.

10. Monique Lebrun et Luc Colles, op. cit., p. 12.

11. Mireille KUTT'TL, La pérégrine, Lausanne, L'Âge d'Homme, 1983.

12. Adrien PASQUALl, Eloge du migrant, Lausanne, Éditions de l'Aire, 1984. 
lointain, qui se confond avec le lieu de sa consécration littéraire, et ce, même si son livre porte spécifiquement sur la vie au sein de cette colonie. L'inverse n'est pas nécessairement vrai pour son vis-à-vis beur qui aurait publié à l'étranger, perdant du coup son droit à la "nationalité» littéraire française ou belge. Il y a là sans doute un autre effet retors de la colonisation, qui explique la relative fermeture de la Belgique, et plus encore de la France, par rapport au "modèle" québécois ou suisse: "Il nous est apparu, au cours de nos travaux, que les écrivains de la migration québécois et suisses avaient une conception de la culture plus ouverte que les autres écrivains migrants de la francophonie" (p. 330). L'explication qu'avancent immédiatement Lebrun et Collès pour exprimer cet état de fait est en soi discutable, puisqu'elle ne tient pas compte de la diversité de l'immigration en France: "Cela vient sans doute à la fois de la diversité de leurs origines (ils ne sont pas majoritairement maghrébins, comme en Belgique, mais de toutes provenances) et de la place qu'on leur fait dans la société" (p. 330). En revanche, l'explication qui suit est plus convaincante dans la mesure où elle précise le sens de cette "place" laissée vacante en France et en Belgique: "Puisqu'il faut parler d'identité, disons que l'écrivain migrant québécois ou suisse vient décentrer le dispositif identitaire de la majorité et lui fait entrevoir une conception pluraliste de la culture, alors que les écrivains migrants français et belges recentrent encore plus le dispositif identitaire de la majorité, certains écrivains migrants jouant le jeu de l'ethnicisme" (p. 331). Ce diagnostic du clivage France-Belgique/ Suisse-Québec, somme toute relatif, reprend la critique pertinente que les auteurs font du modèle hégémonique français en se référant à Bourdieu, pour qui "la littérature est une patrie intemporelle" (p. 329) et "la littérature française [une patrie] plus intemporelle et universelle que les autres, et spécifiquement, plus universelle et intemporelle que les littératures francophones de Belgique, de Suisse et du Québec, entre autres" (p. 329). Or, ils auraient pu en arriver au même constat, plus nuancé toutefois, s'ils s'étaient souvenus que la Suisse n'a jamais eu de colonies... et que le Québec est lui-même une ancienne colonie (et deux fois plutôt qu'une!).

En réalité, deux conceptions de la littérature s'affrontent ici: la conception universalisante, qui est tributaire de la tradition humaniste dans les lettres, la culture et les arts européens, toujours à l'affût du "chefd'œuvre" intemporel, et une conception particularisante, laquelle se déroule en marge de cette tradition en y accueillant la culture populaire, la littérature régionale et les arts mineurs, quel que soit le degré d'achèvement des œuvres. Si la première a triomphé en France, au point d'éclipser la seconde en se "naturalisant" (c'est ce que Barthes nomme une mythologie ou une idéo- 
logie $\left.{ }^{13}\right)$, l'autre a résisté tant bien que mal au sein des grandes puissances (notamment la Grande-Bretagne, l'Allemagne, l'Espagne et l'Italie), avant de trouver dans les petits ensembles ${ }^{14}$ (Pays-Bas, Suisse, Belgique, etc.) un terrain plus fertile pour son épanouissement. Le cas du Québec est symptomatique de ce clivage qu'il a reçu en partage, une soi-disant élite persistant à y célébrer le culte de la littérature universelle, mais coupée de ses racines humanistes qu'elle tente artificiellement de bouturer sur son territoire inculte, tandis que son rebut, la plèbe, continue de ratisser la glèbe qu'on lui a cédée en héritage... Ce que j'avance ici ne plaira pas aux oreilles délicates et sonnera même politiquement très incorrect, mais il se laisse encore vérifier au sujet notamment des écritures migrantes, qui ont reçu leur sanction institutionnelle, certes - et je rappelle que ce mot, "sanction", signifie aussi bien peine que récompense, désaveu que reconnaissance -, mais comme une littérature de seconde zone, au même titre que les littératures régionales, la littérature des femmes, la littérature des minorités sexuelles et la paralittérature. On n’a qu’à consulter les récents manuels littéraires parus au Québec ou qui portent sur sa littérature pour voir comment ces résidus littéraires sont le plus souvent regroupés à la fin, dans un fourre-tout en annexe, comme compléments de la littérature nationale ou canonique.

Ces considérations intempestives, mais non dépourvues d'àpropos, ne sont pas prises en compte par Collès et Lebrun dans un ouvrage qui se veut pourtant pédagogique, comme l'atteste la quatrième de couverture: "Ce livre propose un panorama de la littérature migrante dans l'espace francophone et des pistes d'exploitation pédagogique en classe de français". Luc Collès et Monique Lebrun enseignent d'ailleurs la didactique du français; "langue étrangère et seconde" pour le premier, professeur ordinaire à l'Université catholique de Louvain, "langue première" pour la seconde, professeure titulaire à l'Université du Québec à Montréal. Ensemble, ils ont rédigé le chapitre d'introduction ("La Littérature migrante francophone dans les cursus scolaires»), le chapitre 8 ("Regards croisés Europe-Québec") et la conclusion générale; séparément, ils se sont partagé la litterature migrante de France et de Belgique (Collès: chapitres 2, 3, 4), et la littérature migrante du Québec (Lebrun: chapitres 5, 6, 7). Quant à MarieCécile Robinet, qui est licenciée-agrégée en philologie romane (sans que nous

$$
+4+
$$

13. Roland Barthes, Mythologies, Paris, Seuil, 1957. Voir en particulier l'essai qui suit les a mychologies a à proprement parler: "Le mythe, aujourd'hui".

14. Nous vient immédiacement à l'esprit le très bel essai de François PaRE, Les litteratures de l'exiguitte, Ottawa, Le Nordir, 1992. 
soit précisé son établissement de rattachement), elle a écrit les chapitres 9 et 10 de l'ouvrage concernant la Suisse. Conscients du caractère potentiellement réducteur de leur approche, les auteurs s'en défendent bien dans leur introduction: "Lidentité culturelle comme construction et affirmation par les textes, si elle ne convient pas tout à fait à la critique universitaire, se situe pourtant dans le droit fil de ce que l'école imagine être sa mission culturelle» (p. 16). Mission louable, s'il en fut, encore faudrait-il que le nom des auteurs migrants étudiés en classe soit correctement épelé. C'est le cas le plus souvent dans les études consacrées à l'œuvre de certains écrivains donnés en exemple, mais pas toujours dans l'introduction où l'on retrouve des inconstances navrantes ("Nficone" ou "Nucone" pour Micone, p. 14 et 19; "Lafenière" pour Laferrière, p. 15; "Olivier» pour Ollivier, p. 19), des incongruités confondantes ("Ion Simon" au lieu de Sherry Simon, p. 13), voire des énigmes indéchiffrables (qui est "Bepg" ?, p. 15). Avant de mettre l'ouvrage entre les mains des élèves et des enseignants, il faudrait corriger les nombreuses coquilles qui le parsèment et procéder à sa rapide réimpression.

En ce qui concerne le volet spécifiquement québécois de l'ouvrage, Monique Lebrun s'est attardée à trois corpus distincts: la poésie migrante, avec une étude didactique sur la représentation de la ville chez deux poètes, Anthony Phelps et Mona Latif-Ghattas, et une analyse comparative des poèmes Speak white (Michèle Lalonde) et Speak what (Marco Micone) ${ }^{15}$; l'écriture migrante en relation au territoire, avec une attention particulière accordée aux ouvres de Ying Chen et de Stanley Péan (on devine que ce chapitre a été rédigé plus tôt, puisque la bibliographie des deux auteurs n'a pas été mise à jour); et "l'expérience migrante» de Sergio Kokis (peut-être la contribution la plus originale de l'auteure). Le chapitre qui suit semble encore plus intéressant, car il fait ce qu'à peu près tous les ouvrages consacrés à la littérature migrante omettent de faire: établir un parallèle entre différents corpus "nationaux" (dans ce cas: belge, français et québécois) autour d'une même problématique, bien que réduite ici à un genre (l'écriture des femmes, la dramaturgie migrante et la littérature jeunesse). Rédigées à quatre mains selon une approche résolument comparatiste, ces trois études explorent une voie que l'on aimerait voir plus souvent pratiquée par les spécialistes de la littérature migrante, voire de la littérature tout court. Dommage, en ce sens, que l'étude instructive de Marie-Cécile Robinet portant sur la littérature

$+4+$

15. Cette analyse passe sous silence l'étude de Lise GAUVIN qui accompagne la réédition du poème de Marco MiCONE: Speak what, Montréal, VLB, 2001. 
migrante en Suisse reconduise le modèle obligé de l'annexe ajoutée au corpus principal. Cette étude panoramique aura toutefois eu le mérite d'établir des points de comparaison ponctuels, notamment entre des écrivains suisses et québécois, que les futurs chercheurs pourront reprendre à leur compre afin de poursuivre l'analyse fructueuse des parallèles.

\section{LA TRANSMIGRANCE AU QUÉBEC}

Tout en s'inscrivant dans le sillage de l'ouvrage historique de Moisan et Hildebrand consacré à l'écriture migrante au Québec (1937-1997), qu'il poursuit et élargit au seuil des années 2000 , le panorama didactique que proposent Lebrun et Collès de la littérature migrante dans l'espace francophone opère un retour en arrière sur le plan conceptuel, comme l'indique l'incipit du manuel: «Notre approche de la littérature migrante s'inscrit dans une démarche interculturelle. Celle-ci n'implique pas seulement une ouverture à la culture étrangère, mais exige aussi un véritable travail de retour sur soi, aux sources des origines" (p. 5). En effet, la question du transculturel est relativement peu abordée dans cet ouvrage (à l'exception du chapitre 6 dédié à "L'écrivain migrant et ses pays») au profit de l'interculturel. $\mathrm{Ce}$ changement de perspective s'explique par la visée pédagogique poursuivie par les auteurs, constamment soucieux de mettre en relation non seulement des corpus, mais des élèves provenant de milieux culturels différents. Le «choc des cultures" que présuppose une pensée de la transculture, avant de pouvoir opérer une traversée des cultures ou un dépassement du choc initial, est justement ce que les enseignants tentent d'éviter en entamant le dialogue interculturel avec leurs élèves à travers des œuvres appartenant aux corpus migrant et national. Si cette approche est défendable à l'école (encore qu'il ne faille jamais sous-estimer le choc culturel comme facteur d'apprentissage), elle demeure problématique au niveau universitaire - Collès et Lebrun en ont d'ailleurs convenu -, car elle fait l'impasse sur les avancées théoriques opérées par les spécialistes de la transculture, dont les intellectuels italo-québécois qui ont œuvré au sein de la revue Vice versa (1983-1996).

Parmi les théoriciens de l'écriture migrante au Québec et de la transculture (Pierre Nepveu, Régine Robin, Sherry Simon, Pierre L'Hérault), Simon Harel est sans doute celui qui a poussé le plus loin la réflexion sur les avatars du genre et du concept, proposant de l'intérieur une critique devenue nécessaire des excès auxquels le discours consensuel autour de ces notions avait donné lieu. Depuis le Voleur de parcours jusqu'à son tout récent essai, Braconnages identitaires. Un Québec palimpseste, Harel est maintes fois revenu sur la littérature des communautés culturelles, abandonnant en cours de 
route cette appellation jugée trop «ethniciste" pour embrasser le concept plus neutre d'écriture migrante, avant de remettre en question cette nouvelle étiquette devenue entre-temps une mode, sinon une marque de commerce. Dans Les passages obligés de l'écriture migrante, le théoricien, qui a contribué plus que quiconque à la "valorisation de la migrance, forme euphorique du pluralisme" (p. 70), peut sembler maintenant vouloir retourner sa veste. $\mathrm{Ce}$ serait commettre là une erreur de lecture. Sans prétendre parler au nom de ses collègues, Harel rappelle "que cette euphorie masquait l'espace de rencontre entre identités individuelle et collective» (p. 70). Il s'en prend au concept d'altérité qui, à force d'être convoqué positivement dans les travaux sur l'écriture migrante, a favorisé une célébration des formes de l'identitaire au détriment de l'identité: "Avec le recul, je suis tenté d'y voir plutôt un gommage du symbolique en ce qu'il rompt de manière brutale avec les figures de la filiation" (p. 13).

"L'écriture migrante est devenue un fait de discours. Pour cette raison, elle a acquis une valeur générique - ce qui risque d'en faire, dans le pire des cas, une doxa bien rassurante" (p. 39). Nous n'en sommes pas encore là, mais cet avertissement lucide devrait nous servir de mise en garde contre la tentation de faire de la migrance l'apanage de l'étranger, seul désormais à pouvoir jouir du droit de se déplacer en toute impunité sur l'échiquier territorial. Or Harel est tout aussi critique vis-à-vis du discours nationaliste qui défend les privilèges de la majorité: "À l'identité collective, il faudrait opposer la ruse du minoritaire, la valorisation de la marginalité et de la périphérie. Il faudrait, pour les mêmes raisons, questionner l'identité localisée territorialement au profit d'une identité transnationale» (p. 43). Cette identité transnationale et transculturelle, que j'appellerais pour ma part transmigrante, concerne aussi bien l'autochtone que l'étranger, le soi et l'autre. En réponse aux «littératures de l'exiguïtée de François Paré, Simon Harel argue en faveur des "territoires de la contiguïté" (p. 231), ces lieux particuliers où il devient possible d'effectuer des «braconnages identitaires" de part et d'autre de la frontière imaginaire qui départage les identités. Le moment fort du livre, du moins du point de vue théorique, se situe sans doute au chapitre 3 (" Le lieu: un espace méconnu"), lequel prépare la voie à l'essai suivant en offrant une pensée originale du territoire (bien qu'elle doive à Michel de Certeau et à Shmuel Trigano certains de ses concepts, dont la "demeurance ${ }^{16}$ ") qui

$$
+4
$$

16. Shmuel Trigano, Le semps de lexth, Paris, Payot, 2001. 
valorise la notion de l'habitabilité du lieu ou de l'écoumène ${ }^{17}$. Harel rappelle judicieusement que l'oikos grec ne se laissait pas réduire au domus latin, bien qu'il inclût en son sein l'espace privé de la domesticité, mais qu'il s'étendait à 1 'habitus pris au sens large du terme, à savoir l'extension publique du foyer domestique. À la fois agora et forum, centre et périphérie, l'écoumène permet de concevoir l'espace de l'habitabilité comme un lieu ouvert à la négociation de l'identité. En optant pour le néologisme «situalitén, Harel entend critiquer tout à la fois les notions de territorialité et de diversité, ainsi que le concept d'universalité qui gomme le sentiment d'appartenance à une communauté:

La situalité n'est pas l'incarnation du territoire. Elle ne correspond pas à une mécaphysique des profondeurs et de l'enracinement. La situalité n'est pas l'image d'une naissance à même la cerre, ce qui nous mènerait au discours préjudiciable du "droit de sang", sans oublier les cautions ethniques implicites à toute thèse raciste. Mais ne nous contentons pas non plus d'accueillir l'étranger selon les règles contemporaines de la doxa postmoderne. La situalité n'est pas davantage le constat de la diversité des appartenances territoriales et culturelles. (p. 228)

Les autres chapitres du livre illustrent cette thèse par l'analyse approfondie de l'œuvre de quatre écrivains migrants déjà "visités" par le critique: Naïm Kattan, Régine Robin, Antonio D'Alfonso et Émile Ollivier.

Le principal mérite des derniers essais de Simon Harel consacrés à l'écriture migrante et au territoire est de recadrer le champ conceptuel dans lequel ces réalités, toujours actuelles, doivent désormais être appréhendées. Il ne s'agit plus simplement d'ouvrir les frontières et d'élargir les balises dans un geste néolibéral, mais de configurer autrement l'espace habitable à l'intérieur même de ses limites. En termes deleuzo-guattariens ${ }^{18}$, Harel est davantage intéressé par la phase "reterritorialisante" du processus de la territorialisation que par sa phase "déterritorialisante", qui avait mobilisé, selon lui, toute l'attention critique des promoteurs de la migrance. Encore faut-il ajouter qu'il s'y intéresse dans la mesure où le processus n'est pas encore achevé, quand la deuxième phase reste encore ouverte à la négociation. On trouvera dans ces pages, comme dans celles qui constituent la suite de la nouvelle aventure "migrante" de l'auteur, une pensée forte et originale susceptible d'alimenter toute réflexion sur l'avenir des écritures migrantes au sein de

$$
4+4
$$

17. Augustin BERQUE, Ecoumene. Introduction d̀ l'tude des milieux bumains, Paris, Belin, 2000.

18. Gilles Deleuze ec Félix GuatTari, Mille plateaux, Paris, Minuit, 1980. 
l'espace public et institutionnel de la littérature. Est-ce à dire que l'écriture migrante commande effectivement certains passages obligatoires, qui sont autant de pièges qu'il nous faudra désormais apprendre à contourner? À la fin de son compte rendu perspicace du livre de Simon Harel, Ching Selao se demandait "si la critique de l'engouement des écritures migrantes n'est pas elle-même devenue en quelque sorte un discours normatif ${ }^{19}$ \%. On peut lui emboîter le pas et se demander si "la nouvelle doxa» n'est pas autant du côté de la célébration consensuelle de la littérature migrante que du côté de son dénigrement systématique. À défaut de pouvoir circonvenir la doxa, la voie d'évitement résiderait dans un compromis nuancé, entre l'intérêt soutenu mais élargi accordé à la littérature migrante (Lebrun et Collès) et la vigilance critique requise pour une théorisation adéquate du phénomène (Harel). 